\title{
Functional Outcome of Expert Tibial Intramedulary Nailing For Metaphyseal And Diaphyseal Fractures of Tibia
}

\author{
Lakhpat Yadav ${ }^{1}$, S Srivastava ${ }^{2}$, Rakesh Kumar ${ }^{3}$, Gaurav Garg ${ }^{4}$, \\ Jakir Hussain ${ }^{4}$, Anil K. Sharma ${ }^{4}$ \\ ${ }^{1}$ Senior Resident, Department of Orthopaedics, S.M.S Medical College and Hospital, Jaipur \\ ${ }^{2}$ Senoir Consultant, Department of Orthopaedics, S.D.M Hospital, Jaipur \\ ${ }^{3}$ Medical Officer, Department of Orthopaedics, S.M.S Medical College and Hospital, Jaipur \\ ${ }^{4}$ Asstt. Prof, Department of Orthopaedics, S.M.S Medical College and Hospital, Jaipur
}

\begin{abstract}
:
Introduction : In the field of trauma surgery, fractures of the leg remain the injuries with a higher complication rate. The optimum treatment for fractures of the tibia remains controversial. When the aim is stable fixation with early restoration of function without use of plaster, intramedullary nailing offers an attractive treatment option.Newer tibial nail designs has widened the range of indications for medullary osteosynthesis to include almost every type of fracture. This study was done to evaluate the outcome of use of expert tibial nail in metaphyseal and diaphyseal fractures of tibia.

Materials and methods:This study was conducted on 20 skeletally mature patients with metaphyseal or diaphyseal fractures of the tibia treated with Expert Tibial Nail which is having advanced proximal and distal locking options.

Results: Functional results were evaluated as Excellent, Good, Fair, and Poor on the basis of Johner R, Wruhs $S D$ criteria. Out of 20 patients 14 (70\%) patients had excellent results as they had full function as pretrauma without any residual symptoms. 3 patients (15\%) had good results, $2(10 \%)$ patients had fair results and $1(5 \%)$ patient had poor results.
\end{abstract}

Conclusion: Our results with expert tibial interlocking nailing are encouraging and demonstrate the benefits of new nailing system. We recommend the use of expert tibial nail in metaphyseal and diaphyseal fractures of the tibia.

\section{Introduction}

Rising incidence of high velocity trauma due to motor vehicle accidents accidents usually results in fractures of long bones. The tibia is the most commonly fractured long bone in the body ${ }^{1}$. Due to its location, structural anatomy and sparse anteromedial soft tissue coverage the tibia is exposed to frequent injuries, also open fractures are also common in tibia than any other long bone in the body. ${ }^{2}$

Numerous treatment options exist for treating tibial fractures and good results have been reported with both conservative and surgical methods. To reduce the complications ${ }^{3}$ asssociated with conservative treatment, tendency towards operative management of tibial fractures is in voge. Various operative methods like open reduction and plating, intramedullary nailing and external fixation ${ }^{4,5,6}$ have their own indications, advantages and disadvantages. When the aim is stable fixation with early restoration of function without use of plaster, intramedullary nailing, for internal fixation of diaphyseal and metaphyseal fractures of the tibia, offers an attractive treatment option. ${ }^{4}$ Intramedullary splinting provides the biomechanical principle of interfragmentary compression, support and adaptation to the basic principles of osteosynthesis. Interlocking nail has widened the range of indications for medullary osteosynthesis of tibial shaft fractures to include almost every type of fracture. ${ }^{7}$ The major advancement in intramedullary nailing of proximal tibial fractures was the introduction of modern implants like the Proximal Tibial Nail and Expert Tibial Nail. In these new implants the interlocking bolts can be introduced just below the tibia plateau, enabling the surgeon to treat very proximal tibial fractures with intramedullary nailing. ${ }^{8}$

The Expert Tbial Nail System (ETNS) an intramedullary nailing system is indicated for fractures in the tibial shaft as well as for metaphyseal and certain intraarticular fractures of the tibial head and the pilon tibiale. In addition to the standard static and dynamic locking options, the ETNS features multi directional locking options in the distal and proximal part of the nail. End cap block the most proximal screw creating an angular stable construct. ETNS is having an option for compression at the fracture site (up to $7 \mathrm{~mm}$ ) by inserting one distal locking screw and one proximal dynamic locking screw with a compression screw. 


\section{MMaterials and Methods}

Present study was carried out in a total of 20 patients with metaphyseal or diaphyseal fractures of the tibia presentedto us between September 2012 - May 2013, treated with Expert Tibial Intramedullary Nailing. Ethical committee approval was obtained, and patients were recruited once written informed consent had been provided.

Inclusion criteria were skeletally mature patients with (1) Diaphyseal fractures of tibia(2) Extraarticular metaphyseal fractures of proximal and distal tibia (simple, wedge or complex) (3) segmental fractures of tibia or combination of these. Exclusion criteria were skeletally immature patients, comminuted intraarticular fractures, grade IIIC open fractures.

The implant used was Expert Tibial Intramedullary Nail having advanced nail design and 5 proximal locking options(three unique oblique and two medio-lateral locking options) and 4 distal locking options(Two medio-lateral, one antero-posterior and one additional oblique locking option, placed very distally) .

Patient was positioned supine on the radiolucent table ensuring that the knee of the injured leg can be flexed until at least 90-110 degree and tourniquet was applied. Close reduction was performed manually by axial traction under image intensifier. An incision was given proximally at the distal third of the patella along the patellar ligament down to the tibial tuberosity.

The entry site of the nail was prepared on the ventral edge of the tibial plateau. The entry point was in line with the axis of the intramedullary canal and with the lateral tubercle of the intercondylar eminence in AP view, and was at the ventral edge of the tibial plateau in Lateral view. A guide wire was secured on universal chunk and inserted at an angle of 10 degree to the shaft axis in lateral view. The cannulated awl was placed over the guide wire and the medullary canal was opened using a twisting motion to advance the awl to a depth of approx. 8-10 cm taking care not to open posterior cortex. Decision for reaming were taken per-operatively, and medullary canal was reamed by increasing the reamer size by $0.5 \mathrm{~mm}$. The proximal and distal nail position was checked under image intensifier.

Third generation cephalosporins antibiotics were administered in suitable doses. The limb was elevated on Braun Bohler splint to control postoperative swelling. From first postoperative day the patient was encouraged to do active and assisted hip, knee, ankle and toe movements. The skin sutures were removed after 10-12 days after operation. Non-weight bearing crutch walking was started as early as possible. Protected full weight bearing with or without PTB/PVC brace was started depending upon the pattern of fracture and stability of fixation achieved.

The patients were followed in OPD every 4 weeks for first three visits and thereafter every three months for one year. At each follow up visit patient was assessed both clinically and radiologically. X-ray was taken to note the progress of the union. Clinical examination was done to note the range of movements at the knee, ankle and foot, time taken for fracture union, residual shortening, deformity and any other complaints. criteria. $^{9}$

Results were evaluated as Excellent, Good, Fair, and Poor on the basis of Johner R, Wruhs SD

\section{Results}

There were 16 males and 4 females in our study with an average age of 42.8 yrs ( Range 18 to 72 yrs). Majority of patients sustained fracture both bone leg due to road side accidents (80\%), followed by history of fall. In $19(95 \%)$ cases both tibia and fibula were fractured and in $1(5 \%)$ cases only tibia was fractured. 17 patients $(85 \%)$ were having closed fracture while 3 patients were having open fracture. 9 patients $(45 \%)$ patients operated were having fracture of lower $1 / 3^{\text {rd }}$ of tibia. Five $(25 \%)$ patients were having fractures of proximal $1 / 3^{\text {rd }}$ of tibia. Six (30\%) patients were having fractures of middle $1 / 3^{\text {rd }}$ of tibia.Fractures were classified according to A.O. classification.

\begin{tabular}{|l|l|l|}
\hline Type A- No. of patients & Type B- No. of patients & Type C- No. of patients \\
\hline A $1-11$ & B $1-0$ & $\mathrm{C} 1-1$ \\
\hline A2 -5 & B2 -2 & $\mathrm{C} 2-0$ \\
\hline A $3-1$ & B $3-0$ & $\mathrm{C} 3-1$ \\
\hline
\end{tabular}

Reduction was achieved by closed method in 16 (80\%) cases. Fracture site was opened in $4(20 \%)$ cases to perform interlocking nailing. Additional procedure (bone grafting) was done in one case. Out of 20 cases, dynamization was performed in $3(20 \%)$ cases. Postoperative infection was seen in one patient, which lead to delayed union and fracture united at 26 weeks. Average interval of protected full weight bearing was 3.8 weeks, while average interval of unprotected full weight bearing was 7.5 weeks. Average time taken for clinical union was 13.4 weeks while average duration of radiological union was 16.8 weeks. 

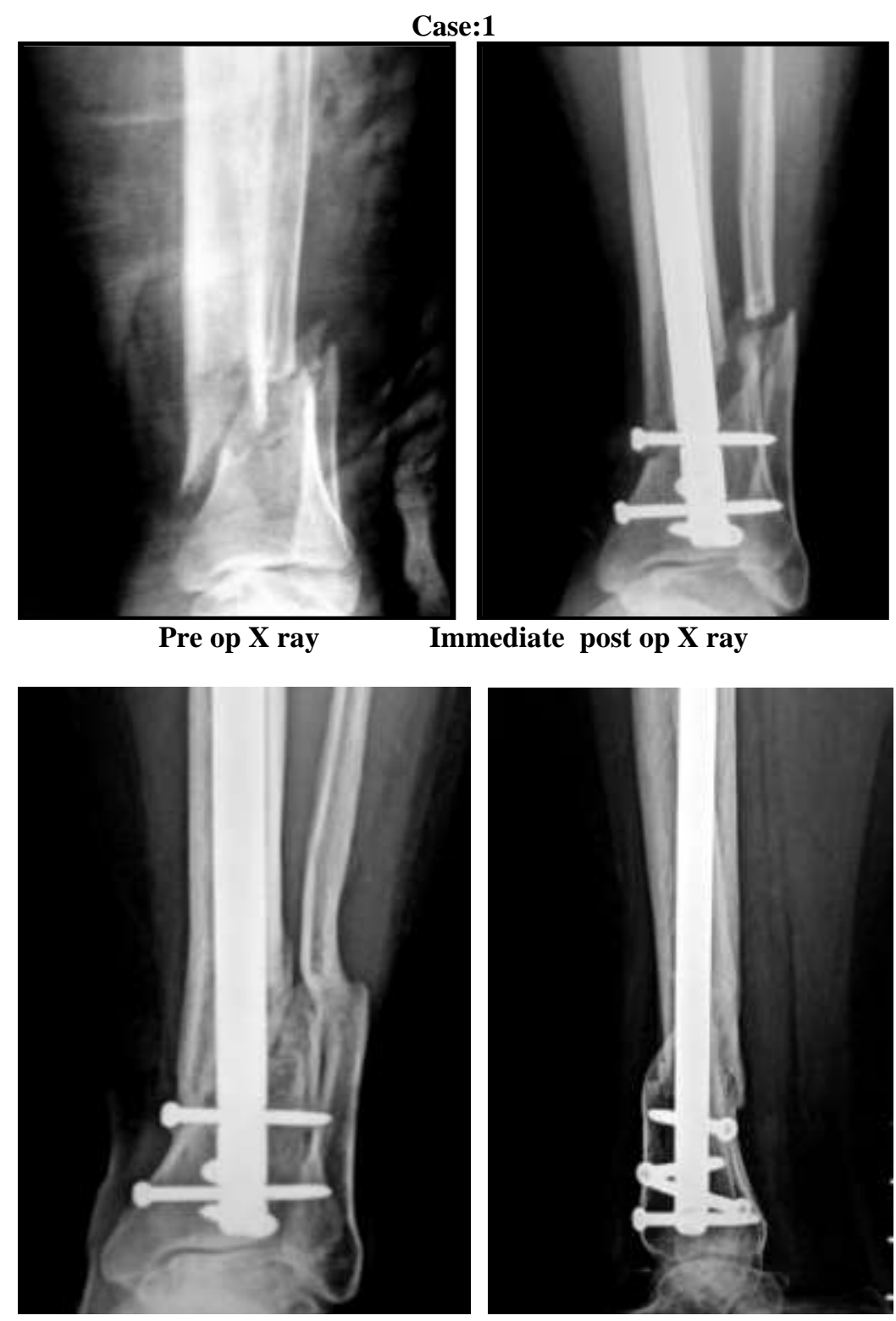

Last follow up X-ray at 5 months showing good consolidation.
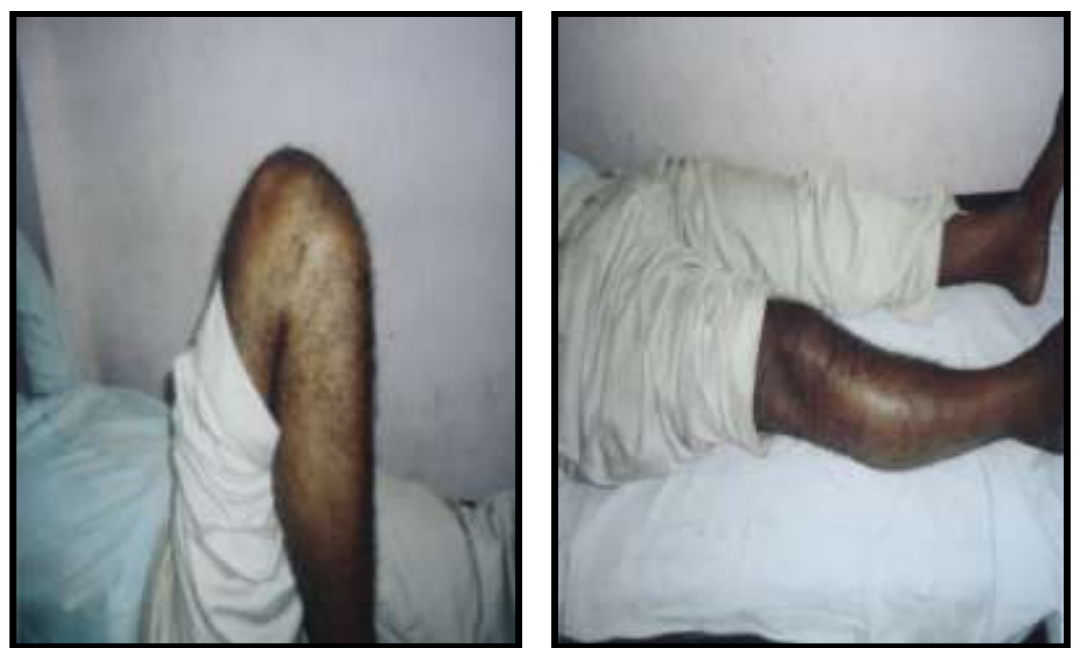

Clinical photograph showing good range of motion in knee 


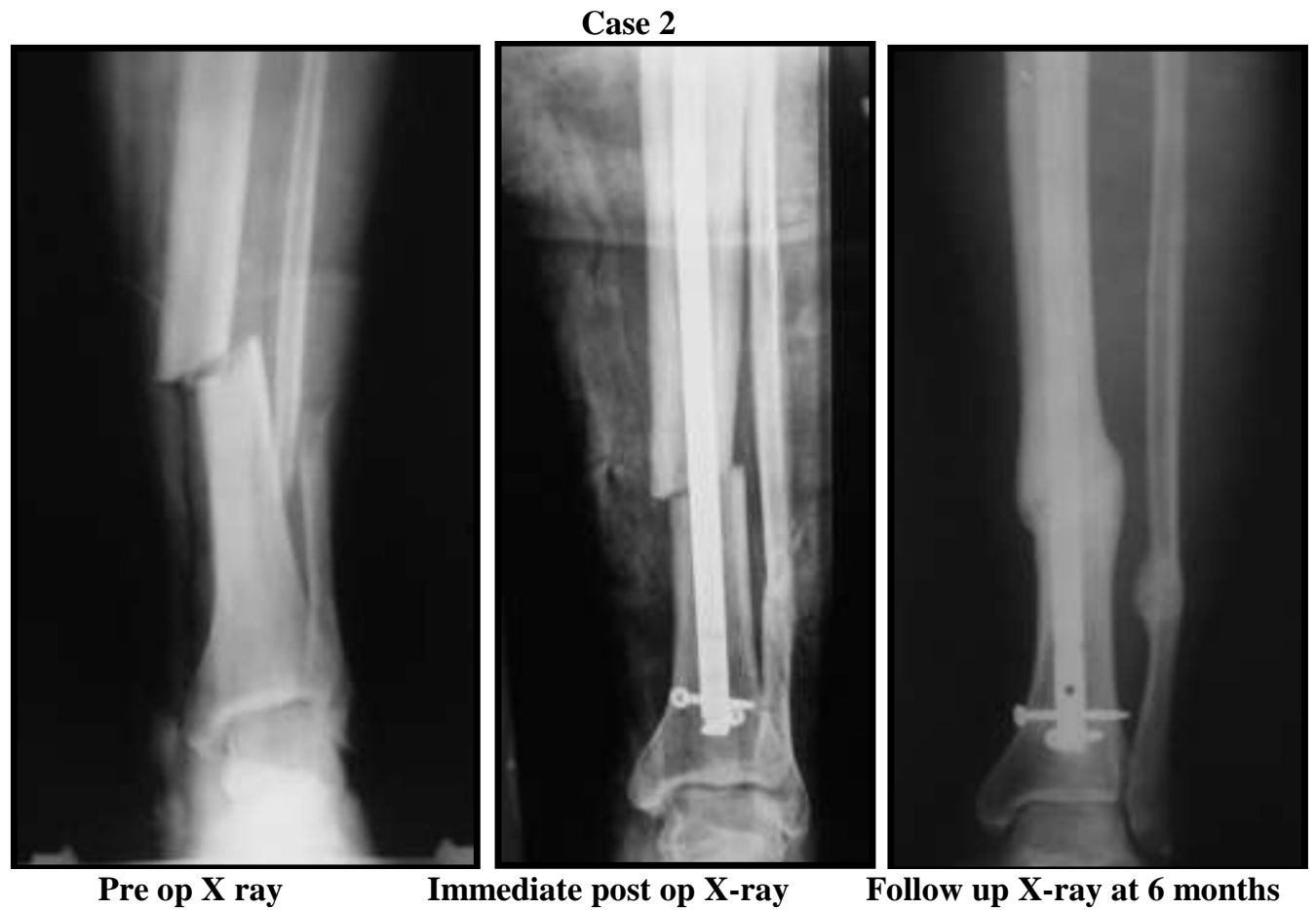

Complications observed in the series were restricted ankle movements in 3 patients, restricted knee movements in 2 patients, valgus at ankle seen in 2 patients, anterior knee pain in 2 patients, delayed union seen in 2 patients.

Results were evaluated as Excellent, Good, Fair, and Poor on the basis of Johner R, Wruhs SD criteria: ${ }^{9}$ Out of 20 patients $14(70 \%)$ patients had excellent results as they had full function as pretrauma without any residual symptoms, 3 patients (15\%) had good results, 2 (10\%) patients had fair results and $1(5 \%)$ patient had poor results.

\section{Discussion}

Fractures of tibia continue to pose vexing problems to the orthopaedic surgeons; chief among them is selecting the optimal method of treatment. Every fracture of tibia must be assessed individually and it can be difficult to establish fixed routine of treatment for these fractures. Internal fixation of diaphyseal and metaphyseal fractures of the tibia with plates may lead to ulceration of skin overlying plate, deep infection leading to osteomyelitis and refracture and delayed union. ${ }^{10}$ Intramedullary nailing offers an attractive treatment option, however there are some problems in treatment of fracture tibia with conventional intramedullary interlocking nailing like difficulty in manipulating fractures of proximal and distal $1 / 3^{\text {rd }}$ tibia communited metaphyseal fractures. These shortcomings of conventional intramedullary interlocking nail in managing proximal and distal third fractures have been overcome by the introduction of Expert Tibial Interlocking Nail due to modifications in operative techniques, its design and advancement in locking screws. ${ }^{8}$

Thus expert tibial nail design allows better control in metaphyseal tibial segments through multiple interlocking holes in close proximity to either end of the nail. Multidirectional interlocking screws ensure that alignment can be well maintained and stability preserved despite a short proximal or distal fragments. We evaluted our results and compared them with those obtained by various other studies.

Mean age in our series was 42.8 years( range 18 to 72 years). Maximum patients were (25\%) in the age of $3^{\text {rd }}$ decade. In the series by Gregory and Sanders ${ }^{11}$ mean age was 30 years while in series of Duwelius et $\mathrm{al}^{12}$ mean age was 40.5 years. Most of the fractures were caused by high-energy trauma, usually as a result of roadside accidents $(80 \%)$. Studies by Gregory and Sanders ${ }^{11}(85.10 \%)$ and Krettek et al ${ }^{13}(71 \%)$ documented similar observations. Maximum no. of cases $9(45 \%)$ were having fracture of tibia in its distal third followed by middle third $6(30 \%)$ and proximal third $5(25 \%)$ type. In a series by Duwelius et al ${ }^{12}$ noted fractures of tibia distal third(45.6\%), middle third $(38.4 \%)$, proximal third $(6.4 \%)$.

Sixteen $(80 \%)$ patients were operated within 10 days of injury. Minimum interval was one day. Blachut et al in their series of 64 fractures have reported the average interval of 22 hours between trauma and injury. ${ }^{14}$ Duwelius et al nailed the closed fractures electively within 24 to 72 hours after trauma. ${ }^{12}$ Gregory and Sanders ${ }^{11}$ operated their patients with an average of 0.9 day after injury. Duration of allowance of weight 
bearing after surgery is determined by personality of fracture, stability of fixation, and also by associated injury. Average duration of protected and unprotected full weight bearing was 4.4 weeks and 7.5 weeks respectively in our series. As most of the fractures in our series were unstable variety we allowed partial weight bearing at 3-4 weeks followed by protected full weight bearing either in PTB cast or PTB-PVC brace. Only when, radiological signs of union were seen full unprotected weight bearing was started. In the series of CJ Hindley et al ${ }^{15}$ and Folleras et $\mathrm{al}^{16}$ average duration of unprotected full weight bearing was 5.6 weeks and 7.4 weeks respectively. Union rate of $90 \%$ is comparable with that published in the literature (In the series of Gregory and Sanders ${ }^{11}$ and Duwelius et $\mathrm{al}^{12}$ union rate was $92 \%$ and $97 \%$ respectively.)

\begin{tabular}{|c|c|c|c|c|}
\hline Complitations & Gregory and Sanders ${ }^{11}$ & ${\text { Duwelius et } \mathrm{al}^{12}}$ & Krettek et $\mathrm{al}^{13}$ & Present series \\
\hline Malunion & $9 \%$ & $9 \%$ & - & $10 \%$ \\
\hline Delayed union & $5 \%$ & $10 \%$ & - & $10 \%$ \\
\hline Infection & $5 \%$ & - & $1.6 \%$ & $5 \%$ \\
\hline Implant failure & $15 \%$ & - & $9.7 \%$ & None \\
\hline
\end{tabular}

\section{Conclusion}

Our results with expert tibial interlocking nailing are encouraging and demonstrate the benefits of new nailing system. Changes in the design of the nail for improved proximal and distal locking enable it to use in proximal and distal metaphyseal fractures of tibia. Contemporary nail designs also include locking options in three planes, which allow a better stabilization of small proximal and distal fragments and obtain a higher stability of the bone implant construct. We recommend the use of expert tibial nail in metaphyseal and diaphyseal fractures of the tibia.

Limitations of our study includes that it was not a comparative study and sample size and follow up period in our series was short. Further studies are required in the future to validate the possible advantages associated with this new nailing system.

\section{References}

[1]. Puno RM, Teynor JT, Nagano J, Gustilo RB. Critical analysis of results of treatment of 201 tibial shaft fractures. Clin Orthop 1986; 212: 113-21.

[2]. Thomas A. Russel, J Charles Taylor, David G, Lavelle. Fractures of the tibia and fibula. In: Rockwood and Green's Fractures in Adults, $3^{\text {rd }}$ Edition. J.B. Lipincott Company, Philadelphia 1991; 2: 1915-82.

[3]. Sarimiento A, Sharpe FE, Embram ZE, Normand P, Shakwiler J. Factors influencing the outcome of closed tibial fractures treated with functional bracing. Clin Orthop 1995; 315: 8-24.

[4]. Lawrence BB, Kenneth DJ. Treatment of tibial fractures by reaming and intramedullary nailing. J Bone Joint Surg 1986; 68A: 87786.

[5]. Wiss DA. Flexible medullary nailing of acute tibial shaft fractures. Clin Orthop 1986; 212: 122-32.

[6]. Hamja KN, Dunkerley GE, Murray MM. Fractures of tibia. A report of fifty patients treated by intramedullary nailing. J Bone Joint Surg 1971; 53B: 696-700.

[7]. Sharan AD, Jeshuran W, Mulligan M, Mc Guire K, Uhl R. Intramedullary nailing in a tibial shaft fracture with distal articular extension. Orthopaedics, 2005; 28(6): 571-2.

[8]. Kuhn S, Hansen M, Rommens PM. Extending the indication of intramedullary nailing of tibial fractures. Eur J Trauma Emerg Surg 2007; 33: 159-69.

[9]. Johner R, Wruhs O. Classification of tibial shaft fractures and correlation with results after rigid internal fixation. Clin Orthop 1983; 178: 7-25.

[10]. Lottes JO, Hill LJ, Key JA. Closed reduction, plate fixation and medullary nailing of fractures of both bones of the leg. J Bone Joint Surg 1952; 34A: 861-73.

[11]. Gregory P, sanders R. The treatment of closed unstable tibial shaft fractures with unreamed interlocking nails. Clin Orthop 1995; 315: 48-63.

[12]. Duwelius PJ, Schmidt AH, Rubinstein RA, Green JM. Non reamed interlocked intramedullary tibial nailing. Clin Orthop 1995; 315 : 104-13.

[13]. Kretteck C, Schandelmaier P, Tscherne H. Nonreamed interlocking nailing of closed tibial fractures with severe soft tissue injury. Clin Orthop 1995; 315: 34-47.

[14]. Blachut PA, O'bren PJ, Meek RN, Brockhryse HM. Interlocking intramedullary nailing with and without reaming for the treatment of closed fractures of the tibial shaft. J Bone Joint Surg (Am) 1997; 79A: 640-46.

[15]. Hindley CJ, Evans RA, Holt EM, Metcalfe JW. Locked intramedullary nailing for recent lower limb fractures. Injury 1990; 21: 23944.

[16]. Folleras G, Alho A, Stromsoe K, Ekeland A, Thoresen BO. Locked intramedullary nailing of fractures of the femur and tibia. Injury $1990 ; 21: 385-8$ 